\title{
Pattern and Outcome of Traumatic Brain Injury in Geriatric Population in Emergency Hospital Mansoura University Samir Mohamed Attia ${ }^{1}$, Mustafa Mahmoud Nabeeh ${ }^{2}$, Hesham Khairy Ismael ${ }^{3}$, Mustafa Magdy Mohamed Hamada*4 \\ Departments of ${ }^{1}$ Vascular Surgery, ${ }^{2}$ Neurosurgery, ${ }^{3}$ Critical Care Medicine, \\ ${ }^{4}$ Emergency Medicine, Faculty of Medicine, Mansoura University \\ *Corresponding author: Mustafa Magdy Mohamed Hamada, Mobile: (+20) 01033664177, E-Mail: mostafahamada180@gmail.com
}

\section{ABSTRACT}

Background: Traumatic brain injury (TBI) is a critical public health and socioeconomic problem throughout the world, making epidemiological monitoring of incidence, prevalence and outcome of TBI necessary. In addition, TBI in elderly patients is a neglected global disease burden. The main cause is fall, followed by motor vehicle accidents. Severe trauma in elderly population imposes a significant health care burden and is associated with substantial morbidity and mortality.

Objective: To determine the pattern and outcome of traumatic brain injury in geriatric population in order to improve the management of geriatric trauma patients at Emergency Hospital Mansoura University.

Patients and methods: This was a prospective observational clinical study, which was conducted on 206 elderly trauma patients presented and admitted to Emergency Hospital Mansoura University (a level one trauma center with about 250,000 visit and 25,000 trauma cases per year) over a year from December 2019 to December 2020.

Results: Polytrauma was the commonest type with only 35\% of the studied cases were isolated head injury. Most cases had multiple lesions (63.6\%) with only $36.4 \%$ of which had single lesion. The majority of cases had no associated injuries, with few cases had scalp injury (16.5\%) and free abdominal fluid of various degrees (27\%). The average period of hospital stay was $10.42 \pm 3.110$, while the average period of ICU stay was $7.75 \pm 4.538$. The average GOS among the studied cases was $4.83 \pm 2.965$ and the majority of which (359\%) died (grade 5).

Conclusion: The current study demonstrated that, TBI in elderly cases is a life threatening condition with a high mortality rate in which falling from height (FFH) was the predominant mode.

Keywords: Emergency Hospital, Geriatric Population, Traumatic Brain Injury.

\section{INTRODUCTION}

Traumatic brain injury (TBI) is defined as an alteration in brain function caused by an external force. TBI can result in physical, cognitive, emotional and behavioral symptoms and outcome can range from complete recovery to permanent disability or death ${ }^{(\mathbf{1})}$. The World Health Organization (WHO) forecasts that by 2030, TBI will become a leading cause of morbidity and mortality worldwide and presents a major worldwide social, health and economic problem. It is estimated that 10 million people are affected annually by $\mathrm{TBI}^{(2)}$.

Injury mechanisms, patient characteristics, and biological sequelae of TBI among elderly (older than 65 years old) are distinct from those of younger individuals (younger than 24 years old) and require a unique approach to clinical management and research (3). Often neglected, elderly TBI patients are going to be an increasing burden to the society with the worldwide aging population (4). Several unique physiologic and clinical factors make the elderly more prone to a TBI with greater disability including greater frailty, chronic health conditions, polypharmacy and poor strength and balance ${ }^{(5)}$.

Following a TBI, damage occurs in two phases, primary and secondary. Primary injury refers to the initial insult to the brain resulting in immediate cellular perturbation or death. This results in neurological deficits dependent upon the type of injury and specific location within the brain as well as vascular damage which results in immediate hemorrhage ${ }^{(6)}$. Secondary injury is a consequence of primary injury and includes changes in the microenvironment, altered cellular metabolism, diminished vascular response, hypoxia, edema, and inflammation. In the context of a geriatric patient, these mechanisms may be enhanced due to the normal physiologic changes that occur with increased age ${ }^{(7)}$.

Unsurprisingly, older adults with TBI experience higher morbidity and mortality. They show slower recovery trajectories and have on average worse functional, cognitive and psychosocial outcomes than do younger patients. Elderly TBI patients also bear higher cost during hospital stay in cost-analysis models due to the complexity of managing TBI in the elderly in the setting of multiple underlying chronic health conditions ${ }^{(\mathbf{8})}$.

The aim of this study was to determine the pattern and outcome of traumatic brain injury in geriatric population in order to improve the management of geriatric trauma patients at Emergency Hospital Mansoura University.

\section{PATIENTS AND METHODS}

This was a prospective observational clinical study which was conducted on 206 elderly trauma patients presented and admitted to Emergency 
Hospital Mansoura University (a level one trauma center with about 250,000 visit and 25,000 trauma cases per year) over a year from December 2019 to December 2020.

Inclusion criteria: Age group above 65 years old, both genders, isolated and polytraumatized patients with TBI, and blunt or penetrating TBI.

Exclusion criteria: Age group less than 65 years old, patients who were dead on arrival, patients who refuse to participate in the study, and patient who leave hospital against medical advice.

\section{Methods:}

The primary survey:

-Resuscitation of the patient using ABCDE approach:

- Airway maintenance and cervical spine immobilization.

- Breathing and ventilation.

- Circulation and control of hemorrhage.

- Disability: neurological status and Glasgow coma scale (GCS).

- Exposure / environmental control.

\section{The secondary survey:}

I) Full history taking: including age, gender, mode and time of trauma, time of arrival and resuscitation.

II) AMPLE history:

$\mathbf{A}=$ Allergies.

$\mathbf{M}=$ Medication currently used .

$\mathbf{P}=$ Past illnesses / Pregnancy .

$\mathbf{L}=$ Last meal.

$\mathbf{E}=$ Events / Environment related to injury.

III) Clinical Examination including:

A. Vital signs: (Blood pressure, Heart rate, Respiratory rate).

B. Local examination: looking for signs of skull fracture and scalp examination.

C. Neurological examination: assessment of conscious level (using Glasgow coma scale: GCS ${ }^{(9)}$ and lateralization signs.

D. Complete general examination: head-to-toe examination to define associated and occult injuries.

\section{IV) Radiological investigations:}

- $\quad$ FAST (Focused Assessment with Sonography for Trauma) using LOGIQ P5ultrasound machine.

- $\quad$ X-ray (chest, pelvis, lumbosacral spine and cervical spine) using shimadzucollimator $\mathrm{r}-20 \mathrm{c}$ $\mathrm{x}$-ray device.
Multislice CT brain by using Toshiba scanner Aquilion Prime TSX-303A (164-MCCTscanner) with reconstruction at $0.5 \mathrm{~mm}$ slice thickness.

V) Laboratory tests:

- Complete blood count, blood grouping and cross matching.

- Coagulation profile (PT, PTT, and INR).

- Liver functions tests (serum creatinine and blood urea nitrogen).

- Kidney functions test (ALT, AST, and total bilirubin).

VI) Outcome is estimated by:

- Hospital mortality.

- Hospital length of stay.

- Intensive care unit length of stay.

- Complications during hospital stay.

- Functional outcome was estimated using Glasgow outcome scale score and Glasgow outcome scale score extended and the post traumatic scores were compared to the patient score before trauma ${ }^{(\mathbf{1 0})}$.

\section{Ethical consideration:}

Study protocol was approved by

Institutional Review Board (IRB) of Faculty of Medicine, Mansoura University. The research objectives were explained to the participants' relatives individually and in groups. The researcher was available throughout the study. Informed written consent was obtained from each participant's relatives sharing in the study. Confidentiality and personal privacy were respected in all levels of the study. The relatives were informed that the participation was completely voluntary. Collected data were not used for any other purpose.

\section{Statistical analysis}

IBM's SPSS statistics (Statistical Package for the Social Sciences) for windows (version 25, 2017) was used for statistical analysis of the collected data. $\mathrm{P}$ (probability) value $<0.05$ was considered statistically significant. Quantitative variables were expressed as mean and standard deviation, median, interquartile range, minimum and maximum as appropriate while categorical variables were expressed as frequency and percentage. Bivariate Correlations were assessed using Pearson's or Spearman's correlation coefficient depending on the nature of data.

\section{RESULTS}

The demographic characteristics and medical history of the studied patients are shown in table 1. 
Table (1): Demographic characteristics and medical history in the studied sample

\begin{tabular}{|c|c|c|c|c|c|}
\hline \multicolumn{2}{|c|}{ All patients $(n=206)$} & Mean + SD & Median & Range & IQR \\
\hline \multicolumn{2}{|c|}{ Age } & $69.18 \pm 2.590$ & 69.00 & $65.00-76.00$ & $67.00-71.00$ \\
\hline \multirow{2}{*}{ Gender } & Male & $35.0 \%(72)$ & & & \\
\hline & Female & $65.0 \%(134)$ & & & \\
\hline \multirow{2}{*}{ Residence } & Rural & $64.1 \%(132)$ & & & \\
\hline & Urban & $35.9 \%(74)$ & & & \\
\hline \multicolumn{2}{|l|}{ DM } & $72.3 \%(149)$ & & & \\
\hline \multicolumn{2}{|l|}{ HTN } & $66.5 \%(137)$ & & & \\
\hline \multicolumn{2}{|l|}{ IHD } & $27.7 \%(57)$ & & & \\
\hline
\end{tabular}

IQR: Interquartile Range, DM: diabetes mellitus, HTN: hypertension, IHD: Ischemic heart disease in table 2 .

The mode and type of trauma, CT brain results and associated injuries in the studied population are shown

Table (2): Mode and type of trauma, CT brain results and associated injuries in the studied sample

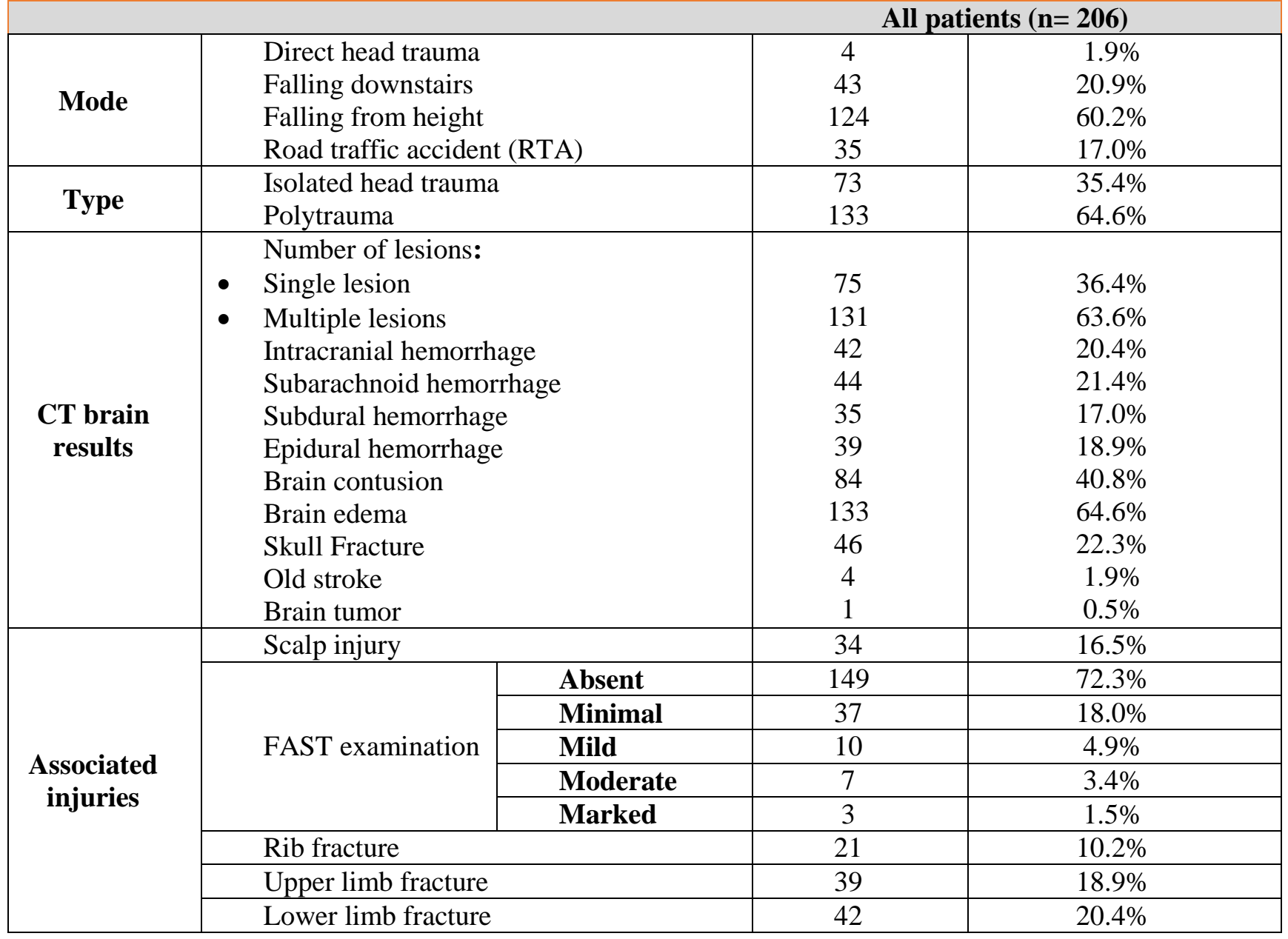

The GCS and the vital signs at admission and length of hospital and ICU stay are shown in table 3.

Table (3): GCS, vital signs at admission and length of hospital and ICU stay (days) in the studied sample

\begin{tabular}{|l|c|c|c|c|}
\multicolumn{1}{|c}{ All patients $(\mathbf{n}=\mathbf{2 0 6})$} & Mean \pm SD & Median & Range & IQR \\
\hline GCS at presentation & $11.17 \pm 2.296$ & 12.00 & $6.00-15.00$ & $9.00-13.00$ \\
\hline Heart rate & $86.68 \pm 11.595$ & 87.00 & $64.00-156.00$ & $78.00-94.00$ \\
\hline $\begin{array}{l}\text { Mean arterial pressure }(\mathbf{m m} \\
\text { Hg) }\end{array}$ & $105.14 \pm 10.031$ & 105.00 & $80.00-137.00$ & $99.00-112.00$ \\
\hline Respiratory rate & $16.19 \pm 3.800$ & 16.00 & $6.00-28.00$ & $14.00-18.00$ \\
\hline Hospital stay (Days) & $10.42 \pm 3.110$ & 10.00 & $3.00-18.00$ & $8.00-13.00$ \\
\hline ICU stay (Days) & $7.75 \pm 4.538$ & 7.00 & $0.00-18.00$ & $4.00-11.00$ \\
\hline
\end{tabular}

GCS: Glasgow coma scale

Glasgow outcome scale in the studied sample is shown in table 4. 
Table (4): Glasgow outcome scale (GOS) in the studied sample

\begin{tabular}{|c|c|c|c|c|c|}
\hline \multicolumn{2}{|c|}{ All patients $(n=206)$} & Mean \pm SD & Median & Range & IQR \\
\hline \multicolumn{2}{|c|}{ GOS } & $4.83 \pm 2.965$ & 6.00 & $1-8$ & $1-7$ \\
\hline \multirow{5}{*}{ Grade } & Death (5) & \multicolumn{2}{|c|}{74} & \multicolumn{2}{|c|}{$35.9 \%$} \\
\hline & Lower moderate disability (4) & \multicolumn{2}{|c|}{3} & \multicolumn{2}{|c|}{$1.4 \%$} \\
\hline & Upper moderate disability (3) & \multicolumn{2}{|c|}{44} & \multicolumn{2}{|c|}{$21.4 \%$} \\
\hline & Lower good recovery (2) & \multicolumn{2}{|c|}{37} & \multicolumn{2}{|c|}{$18.0 \%$} \\
\hline & Upper good recovery (1) & \multicolumn{2}{|c|}{48} & \multicolumn{2}{|c|}{$23.3 \%$} \\
\hline
\end{tabular}

There were statistically significant correlations between mortality and GCS at presentation, Hospital stay as well as ICU stay (Table 5).

Table (5): Correlation between quantitative variables and mortality in the currents study

\begin{tabular}{|l|c|c|}
\hline \multicolumn{2}{|c|}{ Correlation coefficient } & p \\
\hline Age (Year) & 0.025 & 0.723 \\
\hline GCS at presentation & -0.686 & $<0.001$ \\
\hline HR & 0.066 & 0.344 \\
\hline MAP (mm Hg) & 0.026 & 0.712 \\
\hline RR & 0.052 & 0.454 \\
\hline HGB (g/dL) & 0.072 & 0.305 \\
\hline RBC (cells/mcL) & -0.003 & 0.962 \\
\hline WBC (mcL) & 0.106 & 0.131 \\
\hline PLT (mcL) & -0.036 & 0.607 \\
\hline CR ( $\boldsymbol{\mu m o l} / \mathbf{L})$ & 0.003 & 0.961 \\
\hline SGOT (U/L) & -0.077 & 0.274 \\
\hline SGPT (U/L) & -0.083 & 0.236 \\
\hline Na (g/L) & 0.057 & 0.414 \\
\hline K (mmol/L) & 0.093 & 0.185 \\
\hline INR & -0.069 & 0.326 \\
\hline Hospital stay (Days) & 0.479 & $<0.001$ \\
\hline ICU stay (Days) & 0.770 & $<0.001$ \\
\hline
\end{tabular}

\section{DISCUSSION}

In the current study, the average age of the studied cases was $69.18 \pm 2.590$ with male to female $(\mathrm{M} / \mathrm{F})$ ratio of $35 / 65$. The majority of the studied cases were living in rural areas (64.1\%) with only $35.9 \%$ of which were living in urban areas. In addition, most cases were complaining of DM (72.3\%) and HTN $(66.5 \%)$, while only $27.7 \%$ of which were complaining of ischemic heart disease (IHD).

In agreement, Mosenthal et al. ${ }^{(11)}$ revealed that, falls was the most common cause of TBI in elderly cases $(65 \%)$ followed by road traffic accidents (RTA) $(33 \%)$ and lastly assaults (2\%). In addition, Utomo et al. ${ }^{(12)}$ revealed that, of the 428 isolated, older adult TBI cases, the majority were the result of a fall $(88 \%)$, male $(55 \%)$, and aged $>74$ years $(76 \%)$. Furthermore, Peeters et al. ${ }^{(13)}$ demonstrated that, fall and read traffic accident (RTA) were the two most frequent causes of TBI, with falls being reported more frequently than RTA.

As regards, CT brain results, the majority of the studied cases demonstrated multiple lesions $(63.6 \%)$ in which brain edema (64\%) and contusions $(40 \%)$ were considered the main presenting manifestations, followed by skull fractures, subarachnoid hemorrhage (SAH), intracranial hemorrhage ( $\mathrm{ICH})$ and lastly subdural hematomas (SDH) and epidural hematoma (EDH). Currently, a CT scan of the head is the most common diagnostic tool to evaluate acute TBI in the elderly. It can reveal traumatic intracranial injuries such as contusions, brain lacerations, and hemorrhage leading to the formation of hematoma in the extradural, subarachnoid, subdural, or intracerebral compartments within the head. The American College of Emergency Physicians recommends a head CT scan for any patient age 65 years or older who presents with mild head injury $(14,15)$.

The current study came in accordance with a research which was conducted by Adatia et al. ${ }^{(16)}$ and demonstrated that, progression of cerebral contusions occurs in up to $75 \%$ of patients with TBI whatever the age and this contributes to subsequent clinical deterioration and requirement for surgical intervention. On the contrary, Ushewokunze et al. ${ }^{(17)}$ demonstrated that, acute subdural hematoma was the commonest pathology for elderly TBI, which corresponds with a coroner's report that subdural hematoma was the commonest lesion of the deceased elderly persons with head injuries ${ }^{(\mathbf{1 8})}$.

As regards GCS, the current study demonstrated that, the average GCS at admission was 
$11.17 \pm 2.296$. Such average came in the majority of the studied cases who were demonstrated that, most cases demonstrated mild TBI with GCS average of 13 to $15^{(19,20)}$.

In terms of mortality, the percentage of mortality among the studied cases was demonstrated to be $35.9 \%$ (74 cases). When considering mortality after geriatric TBI, it is important to distinguish between short-term mortality (during initial hospitalization or rehabilitation) and longer-term mortality (over months or years after TBI). Short-term mortality post-TBI is high among older adults, particularly those with severe TBI, with several studies reporting in-hospital mortality rates as high as $70-80 \%$ in this population ${ }^{(\mathbf{2 1} \text {, }}$ 22).

Medical complications can arise from concurrent injuries, co-morbidities, frailty, previous trauma and drug-drug interactions. Pre-existing diseases which predicted greater mortality post-TBI have been reported to be cancer, kidney disease, liver disease and heart and lung disease ${ }^{(\mathbf{1 9}, \mathbf{2 3})}$.

This came in agreement with McIntyre et al. (19), who conducted their study on twenty-four studies had an overall mortality rate of $38.3 \%$ (CI 27.1$50.9 \%$ ). The odds of mortality for those over 75 years compared to those of 65-74 years was 1.734 (CI= $1.311-2.292 ; \mathrm{p}<0.0001)$. Pooled mortality rates for mild (GCS 13-15), moderate (GCS 9-12) and severe (GCS 3-8) head injuries were $12.3 \%$ (CI $=6.1$ $23.3 \%), 34.3 \%(\mathrm{CI}=19.5-53.0 \%)$ and $65.3 \%(\mathrm{CI}=$ 53.1-75.9), respectively. Odds ratios comparing severe to mild and moderate to mild head injuries were $12.69(\mathrm{CI}=5.29-30.45 ; \mathrm{p}<0.0001)$ and $5.31(\mathrm{CI}=$ $3.41-8.29 ; \mathrm{p}<0.0001)$, respectively. There was no significant difference in the odds of death between severe and moderate injuries $(\mathrm{p}=0.116)$.

Similarly, Mosenthal et al. ${ }^{(24)}$ revealed that the mortality rate after TBI in elderly cases was $30 \%$. On the contrary, Prasad et al. ${ }^{(25)}$ reported lower mortality incidence as they demonstrated that there were seven deaths $(9.5 \%)$ following traumatic brain injury in the elderly in their study. The rate of poor outcome was $26 \%$ overall and $45 \%$ in patients age $\geq 75$ years. Moreover, Grossman et al. ${ }^{(26)}$, displayed that, mortality rates following TBI in old age cases was $8 \%$ only, which came in disagreement with the current study. In addition, Røe et al. (27) conducted a prospective, national multicenter study including patients with severe TBI $\geq 65$ years. They demonstrated that, seven patients, mean age 75 (SD 7) years, $64 \%$ men, were included. Two patients were lost to follow-up; 48 died within 14 days. The predicted versus the observed odds ratio (OR) for mortality was 2.65. Unfavorable outcome (GOSE < 5) was observed at one year follow-up in $72 \%$ of patients.

As regards the average period of hospital stay, it was $10.42 \pm 3.110$ and the average period of ICU stay was $7.75 \pm 4.538$. In accordance to some extent Levant et al. ${ }^{(28)}$ who reported that, the average length of stay for inpatients with a first-listed diagnosis of TBI with a stay in the ICU was 7.3 days, with an average of 1.2 days in the ICU. The average length of stay for inpatients hospitalized for TBI without a stay in the ICU was 3.2 days.

As regards, Glasgow outcome scale (GOS), the average value was $4.83 \pm 2.965$. In addition, the majority of access died (35.9\%), 21.4\% developed Upper moderate disability, while $23.3 \%$ developed Upper good recovery, $18 \%$ developed Lower good recovery and $1.4 \%$ developed Lower moderate disability. In accordance, Chamoun et al. (29) demonstrated that, at the 6-month follow-up, $13.2 \%$ of the entire series achieved a good functional outcome ([GOS] score of 1 or 2). While, Kristman et al. ${ }^{(30)}$ demonstrated that $80 \%$ of the studied cases demonstrated score of 4-5 and less than 20\% demonstrated score less than 4. In addition, Corral $\boldsymbol{e t}$ al. ${ }^{(31)}$ demonstrated that, outcome (GOS and GOSE at 6 months and 1 year) was better in the high GCS score at admission (6-8) group than in the low score group (3-5). The improvement in GOS scores between 6 months and 1 year was greater in the high GCS score at admission group than in the low score group. At 6 months, 75 patients had died and 120 survived. None died between the 6-12-month assessments; at 12 months, $36 \%$ had improved GOS score.

Generally, it is well established that, on average, older adults with TBI have higher mortality $(19,32)$, slower rates of functional and cognitive recovery (24, 33) and worse functional outcomes post-TBI compared to their younger counterparts ${ }^{(8)}$.

\section{CONCLUSION}

The current study demonstrated that, TBI in elderly cases is a life threatening condition with a high mortality rate in which FFH was the predominant mode. In addition, ICU stay, hospital stay, GOS and GCS at presentation were considered main contributing factors for mortality among elderly cases.

\section{RECOMMENDATIONS}

- Rapid as well as prompt treatment in elderly cases with TBI is of great importance.

- Treatment of associated injury on line with treatment of elderly to prevent disabilities and complications.

- Further studies are needed in the future with further subdividing the cases into two groups (above 75 and below 75) to evaluate the role of age accurately.

- Further studies are needed in the future with further emphasizing on the role of associated medical conditions.

- GOS must be applied for every TBI.

- GOS is the best score for determination and prediction of TBI cases. 


\section{REFERENCES}

1. Menon D, Schwab K, Wright D et al. (2010): Position statement: definition of traumatic brain injury. Archives of Physical Medicine and Rehabilitation, 91(11): 1637-1640.

2. Roozenbeek B, Maas A, Menon D (2013): Changing patterns in the epidemiology of traumatic brain injury. Nature Reviews Neurology,9(4): 231-36.

3. Gardner R, Dams-O'Connor $\mathrm{K}$, Morrissey $\mathrm{M}$ et al. (2018): Geriatric traumatic brain injury: epidemiology, outcomes, knowledge gaps, and future directions. Journal of Neurotrauma, 35(7): 889-906.

4. Mak C, Wong S, Wong G et al. (2012): Traumatic brain injury in the elderly: is it as bad as we think? Current Translational Geriatrics and Experimental Gerontology Reports, 1(3): 171-178.

5. Krishnamoorthy $\mathrm{V}$, Distelhorst $\mathrm{J}$, Vavilala $\mathrm{M}$ et al. (2015): Traumatic brain injury in the elderly: burden, risk factors, and prevention. Journal of Trauma Nursing, 22(4): 204-208.

6. Prakash R, Carmichael S (2015): Blood-brain barrier breakdown and neovascularization processes after stroke and traumatic brain injury. Current opinion in Neurology, 28(6): 556-63.

7. Wilberger J, Jho D (2014): Geriatric neurotrauma. In Geriatric Trauma and Critical Care. Springer, New York, NY. Pp. 237-248.

8. Williams H, Caplan B, Bogner J et al. (2015): Epidemiology of adults receiving acute inpatient rehabilitation for a primary diagnosis of traumatic brain injury in the United States. Journal of Head Trauma Rehabilitation, 30(2): 122-135.

9. Dash H, Chavali S (2018): Management of traumatic brain injury patients. Korean Journal of Anesthesiology, 71(1): $12-21$.

10. McMillan T, Wilson L, Ponsford J et al. (2016): The Glasgow outcome scale-40 years of application and refinement. Nat Rev Neurol., 12: 477-485.

11. Mosenthal A, Lavery R, Addis $M$ et al. (2002): Isolated traumatic brain injury: age is an independent predictor of mortality and early outcome. Journal of Trauma and Acute Care Surgery, 52(5): 907-911.

12. Utomo W, Gabbe B, Simpson $P$ et al. (2009): Predictors of in-hospital mortality and 6-month functional outcomes in older adults after moderate to severe traumatic brain injury. Injury, 40(9): 973-977

13. Peeters W, van den Brande $R$, Polinder $S$ et al. (2015): Epidemiology of traumatic brain injury in Europe. Acta Neurochirurgica, 157(10): 1683-1696.

14. Jagoda A, Bazarian J, Bruns J et al. (2009): Clinical policy: neuroimaging and decision making in adult mild traumatic brain injury in the acute setting. Journal of Emergency Nursing, 35(2): 5-40.

15. Tracy $T$, Menaker J, Gambert $S$ et al. (2010): Traumatic brain injury in the elderly. Clinical Geriatrics, 18(4): 20-24.

16. Adatia $K$, Newcombe V, Menon $D$ (2020): Contusion progression following traumatic brain injury: a review of clinical and radiological predictors, and influence on outcome. Neurocritical Care, 1: 1-8.

17. Ushewokunze S, Nannapaneni $R$, Gregson $B$ et al. (2004): Elderly patients with severe head injury in coma from the outset--has anything changed? British Journal of Neurosurgery, 18(6): 604-607.

18. Chisholm K, Harruff $\mathbf{R}$ (2010): Elderly deaths due to ground-level falls. The American Journal of Forensic Medicine and Pathology, 31(4): 350-354.

19. McIntyre A, Mehta S, Aubut J et al. (2013): Mortality among older adults after a traumatic brain injury: a metaanalysis. Brain Injury, 27(1): 31-40.

20. Cheng $P$, Lin $H$, Lee $Y$ et al. (2014): Higher mortality rates among the elderly with mild traumatic brain injury: a nationwide cohort study. Scandinavian Journal of Trauma, Resuscitation and Emergency Medicine, 22(1): 7-13.

21. Mitra B, Cameron $P$, Gabbe B et al. (2008): Management and hospital outcome of the severely head injured elderly patient. ANZ Journal of Surgery, 78(7): 588-592.

22. Brazinova A, Mauritz $W$, Leitgeb $J$ et al. (2010): Outcomes of patients with severe traumatic brain injury who have Glasgow Coma Scale scores of 3 or 4 and are over 65 years old. Journal of Neurotrauma, 27(9): 1549-1555.

23. Lane P, Sorondo B, Kelly J (2003): Geriatric trauma patients-are they receiving trauma center care? Academic Emergency Medicine, 10(3): 244-250

24. Mosenthal A, Livingston D, Lavery $R$ et al. (2004): The effect of age on functional outcome in mild traumatic brain injury: 6-month report of a prospective multicenter trial. J Trauma, 56:1042-1048.

25. Prasad G, Anmol N, Menon G (2018): Outcome of traumatic brain injury in the elderly population: a tertiary center experience in a developing country. World Neurosurgery, 111: 228-234.

26. Grossman M, Miller D, Scaff D et al. (2002): When is an elder old? Effect of preexisting conditions on mortality in geriatric trauma. Journal of Trauma and Acute Care Surgery, 52(2): 242-246.

27. Røe C, Skandsen T, Manskow U et al. (2015): Mortality and one-year functional outcome in elderly and very old patients with severe traumatic brain injuries: observed and predicted. Behavioural Neurology, 2015: 845491.

28. Levant S, Chari K, DeFrances C (2016): National Hospital Care Survey Demonstration Projects: Traumatic Brain Injury. National Health Statistics Reports, (97): 1-16.

29. Chamoun R, Robertson C, Gopinath $S$ (2009): Outcome in patients with blunt head trauma and a Glasgow Coma Scale score of 3 at presentation. Journal of Neurosurgery, 111(4): 683-687.

30. Kristman V, Brison R, Bédard M et al. (2016): Prognostic markers for poor recovery after mild traumatic brain injury in older adults: a pilot cohort study. The Journal of Head Trauma Rehabilitation, 31(6): 33-43.

31. Corral L, Ventura J, Herrero J et al. (2007): Improvement in GOS and GOSE scores 6 and 12 months after severe traumatic brain injury. Brain Injury, 21(12): 1225-1231.

32. Ramanathan D, McWilliams N, Schatz $P$ et al. (2012): Epidemiological shifts in elderly traumatic brain injury: 18year trends in Pennsylvania. Journal of Neurotrauma, 29(7): 1371-1378.

33. Frankel J, Marwitz J, Cifu D et al. (2006): A follow-up study of older adults with traumatic brain injury: taking into account decreasing length of stay. Archives of Physical Medicine and Rehabilitation, 87(1): 57-62. 